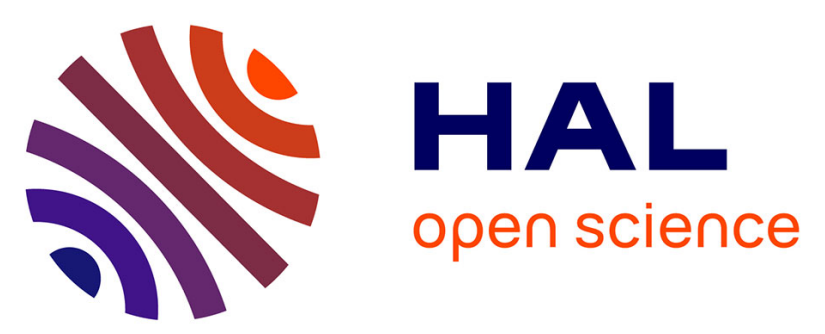

\title{
A design and evaluation framework for a tele-immersive mixed reality platform
}

Simon Crowle, Michael Boniface, Benjamin Poussard, Stylianos Asteriadis

\section{To cite this version:}

Simon Crowle, Michael Boniface, Benjamin Poussard, Stylianos Asteriadis. A design and evaluation framework for a tele-immersive mixed reality platform. 1st International Conference on Augmented and Virtual Reality, Sep 2014, Lecce, Italy. pp.151-158, 10.1007/978-3-319-13969-2_12 . hal-01186353

\section{HAL Id: hal-01186353 \\ https://hal.science/hal-01186353}

Submitted on 22 Sep 2017

HAL is a multi-disciplinary open access archive for the deposit and dissemination of scientific research documents, whether they are published or not. The documents may come from teaching and research institutions in France or abroad, or from public or private research centers.
L'archive ouverte pluridisciplinaire HAL, est destinée au dépôt et à la diffusion de documents scientifiques de niveau recherche, publiés ou non, émanant des établissements d'enseignement et de recherche français ou étrangers, des laboratoires publics ou privés. 


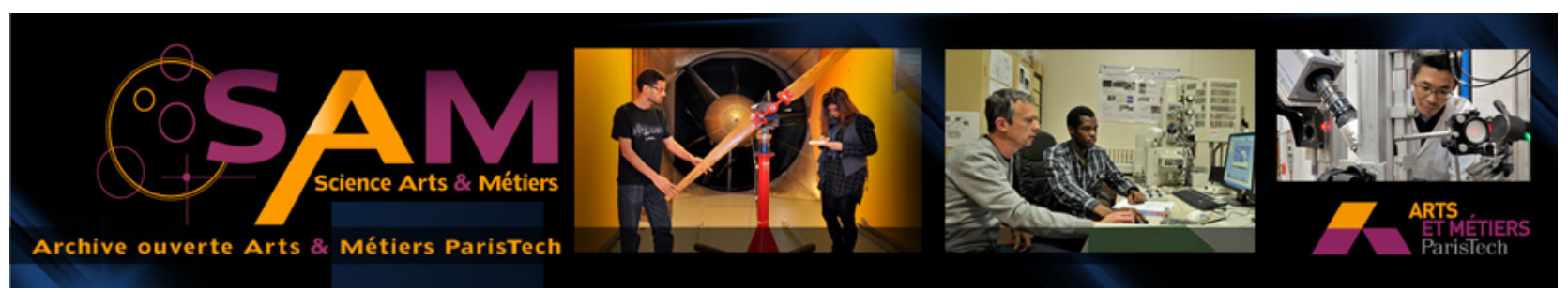

\section{Science Arts \& Métiers (SAM)}

is an open access repository that collects the work of Arts et Métiers ParisTech researchers and makes it freely available over the web where possible.

This is an author-deposited version published in: http://sam.ensam.eu

Handle ID: .http://hdl.handle.net/10985/9855

\section{To cite this version :}

Simon CROWLE, Michael BONIFACE, Benjamin POUSSARD, Stylianos ASTERIADIS - A design and evaluation framework for a tele-immersive mixed reality platform - In: Salento AVR 2015 1st International Conference on Augmented and Virtual Reality, Italie, 2014-09-17 - Augmented and Virtual Reality - 2014 


\title{
A design and evaluation framework for a tele-immersive mixed reality platform
}

\author{
Simon Crowle ${ }^{1}$, Michael Boniface ${ }^{1}$, Benjamin Poussard ${ }^{2}$, Stylianos Asteriadis ${ }^{3}$ \\ ${ }^{1}$ IT Innovation Centre, University of Southampton, SO16 7NS, U.K. \\ sgceit-innovation.soton.ac.uk \\ 2 ARTS Presence \& Innovation Team, Ingénierium - 4, \\ Rue de l'Ermitage - 53000, Laval, France \\ benjamin.poussardeensam.eu \\ 3 Information Technologies Institute, \\ Centre for Research and Technology Hellas, Greece \\ stiasteiti.gr
}

\begin{abstract}
Tele-immersive, mixed reality interactive systems bring remote users together to share a common experience in an environment that synthesizes aspects of the real and virtual worlds. The 3D-LIVE platform is an example of one such system that synthesizes 3D models, audio, motion capture and activity recognition from a number of geographically separated sources into a single gaming environment to support a variety of sports based activities. The design and evaluation of such systems is challenging since factors relating to the technical quality of service (QoS) and quality of experience (QoE) are difficult to identify and measure. In this paper we present a novel QoS/QoE model and evaluation methodology that is being used in the development and testing of the 3D-LIVE mixed reality platform. Our initial results provide some insights into the quality of user experience (UX) we observed from users interacting with 3D-LIVE and are evaluated in the light of QoS data captured. We conclude by discussing the impact of these findings on future platform developments.
\end{abstract}

Keywords: teleimmersion, mixed reality, quality of experience, quality of service, co-creation

\section{Introduction}

Contemporary consumers' computing technologies found at home or in mobile devices are typically capable of providing rich user interfaces that offer virtual or augmented reality presentations. In the computer games market, these experiences are enriched through shared collaborative or competitive game play with friends connected via the Internet. A range of interactive modalities including virtual reality (VR); augmented reality (AR); voice based group communication; and body motion tracking are now integrated in popular, modern computer game design. The 3D-LIVE project [1] is developing a platform for real-time, interactive teleimmersion that connects augmented, interactive experiences situated in real-world activities with correspond- 
ing, simulated activities realised in virtual environments elsewhere. Our goal is to generate a shared sense of presence, co-location and engagement between all users taking part in mixed reality sporting activities and to gain insight into what design and technical considerations must be taken into account to realise this UX. In the following sections we present a high level overview of the UX driven design process and model based approach that is being used to progressively refine, improve and evaluate the 3D-LIVE system. Following this, we present results from our preliminary evaluation work and discuss how the early findings have helped direct prototype developments for enhanced UX.

\section{Background}

Mixed reality teleimmersion is a progression of conventional 3D virtual environments through interaction with the inclusion of full body motion capture and augmented reality presentation technology. Unlike more conventional systems, mixed reality platforms necessarily include real world contexts that introduce inherently more complex and distributed interactions that are challenging both technically and also in terms of understanding their impact on UX [2][6]. Technology used in an attempt to increase the user's perception of full tele-immersion includes the use of CAVE based environments [3], full body motion capture and reconstruction [4] [17] and mobile, augmented reality applications [5]. Variations of traditional sporting activities have been explored using mixed reality technologies. Sports games have either been replicated in a virtualised form [7] [8] or redesigned for remote interaction and training [9] [10].

In 3D-LIVE, we extend this work by exploring the realisation of three sports related scenarios (golfing, jogging, skiing) that engage users situated both outdoors and indoors. In the real world, outdoor users interact via augmented reality presentations on a mobile device and wearable sensors. Two deployments exist for indoor users using virtual reality, in order to assess the impact of different configurations on UX: a 'high end' configuration which includes a CAVE environment, wearable motion sensors, dedicated simulators and rendering of full-body reconstruction of remote users [17]. The consumer level or 'low end' configuration displays the virtual environment on a large, conventional display and integrates Nintendo's Wii for simulation, while simple avatars represent the remote users. In both cases, Microsoft's Kinect depth sensors are used to capture motion, 3D information of user silhouette and evaluate activity using fuzzy engines [20]. A typical test case for a 3D-LIVE scenario is to include one outdoor user with two indoor (but geographically separate) users. An outdoor user is connected to indoor users using a mobile application that operates as a sensor gateway and voice communication channel. Outdoor human activity is acquired by a combination of inbuilt mobile device sensors (e.g. GPS position, direction) and wearable inertia-based wearable sensors (e.g. motion analysis). Local environment data is acquired by a combination of wearable environment sensors that is aggregated with in-situ weather sensors and wide area weather services. All data is captured and communicated in real time to a $3 \mathrm{D}$ virtual environment for rendering to 
the indoor users. Each indoor sees himself as a 3D avatar in the virtual environment along with the other players; his direction of travel is captured using a combination of motion capture sensors and activity recognition algorithms. Each scenario is based on an interaction design that aims to enhance UX associated with distant collaboration within the context of shared sporting activities.

- Golf (Laval, France): Players collaborate in a 'scramble play' variation of the golfing game in which indoor and outdoor players take shots towards the hole and then select the ball judged to be in the most favorable position for the next stroke.

- Jogging (Oulu, Finland): The outdoor jogger initially leads a short jogging route around streets in Oulu whilst the indoor users follow. The aim is for all runners to keep together as a group and achieve the best possible time for the route.

- Skiing (Schladming, Austria): Players race down a slalom course led by the outdoor skier. The slalom course is positioned on the real and virtual slopes. The outdoor skier leads the group down the slope whilst the indoor users attempt to keep up by following their path.

Fig. 1. Golfing scenario indoor golf simulator (VR) and outdoor view (AR)

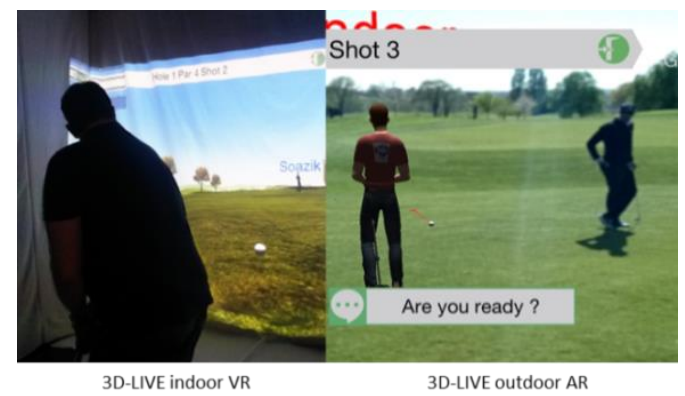

\section{3D-LIVE design process and evaluation model}

3D-Live uses an iterative process of user-driven design and prototype based evaluation to develop an understanding of the necessary interaction design and technical characteristics that combine to deliver a compelling UX. Engagement of users throughout the development life-cycle using scenario based approaches is a wellestablished design practice for VR design [15][14]. Specialisations of these approaches that introduce a formal model to represent system behaviour and UX have been created to evaluate usability experimentally [16]. We build on this work by instantiating an 'experiential model' that includes concepts that are used formatively in the design process as a structure for engaging user groups in 'co-creation activities' associated with the real-world contexts of scenarios. The outcomes allowed us to update the experiential model based on users' views experiential priorities and to direct ongoing design and implementation of the system itself.

From co-creation activities and research work about UX, we built the 3D-LIVE Immersive User eXperience (IUX) model. IUX can be described by two engines: The 
Rational Engine, and the Experiential engine (see Fig. 2). The Rational engine mainly reuses the DIME (Distributed Interactive Multimedia Environment) indicators of UX [16]. It includes the three following elements: Psychological Flow, Telepresence and Technology Acceptance. The Experiential engine is based on emotion and intuition as described in the CEST (Cognitive Experiential Self Theory) [18], and includes the following constructs: Social Presence, Social Emotion, and Emotional Response. The QoS constructs cover the technological aspects of the platform and are derived from DIME's QoS model. Among those constructs are Interactivity (Speed, Range), Vividness (Breadth, Depth) and Consistency (Temporal, Spatial). A model of QoS and QoE that is aligned with the IUX constructs are required to evaluate UX.

Fig. 2. Immersive UX Constructs aligned with Influential Factors and Process Layers (UX Model extended from Wu et al., 2009)

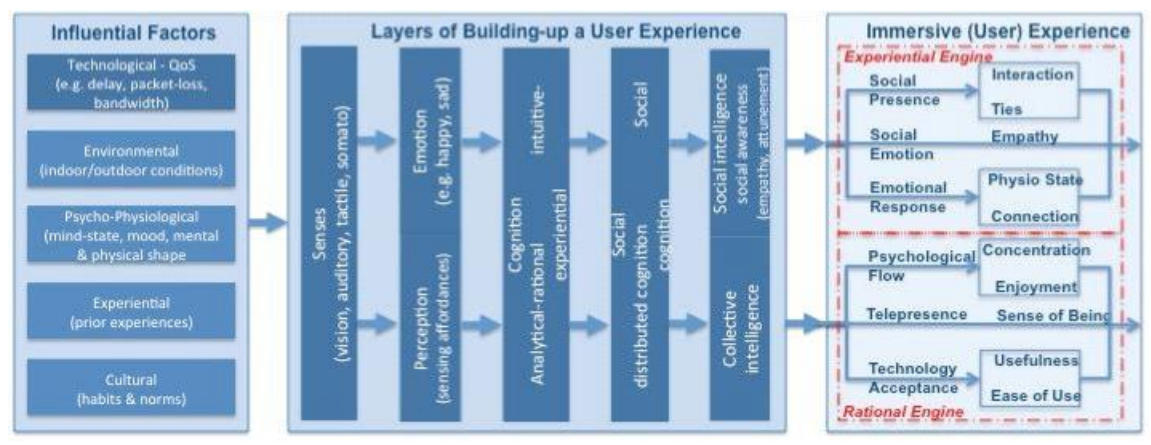

Applying this model starts from identifying the specific metric set representative of the UX to be assessed. Users have been involved in the iterative design process for the three scenarios (Golfing, Jogging, and Skiing). User needs and requirements have been gathered to define the set of metrics related to the constructs of the QoE/QoS table, as shown by two example metrics on the Table 1, to assess the experience they do expect, and what is really important for them.

Table 1. Example sets of metrics for corresponding 3D-LIVE QoE/QoS constructs

\begin{tabular}{|l|l|l|l|l|}
\hline Dimensions & Criteria & Constructs & Metrics & Nature \\
\hline QoS & Consistency & Spatial & $\begin{array}{l}\text { Path Accu- } \\
\text { racy of the } \\
\text { outdoor } \\
\text { avatar }(\mathrm{m})\end{array}$ & $\begin{array}{l}\text { Log: GPS positions } \\
\text { compared to avatar } \\
\text { position }\end{array}$ \\
\hline QoE & $\begin{array}{l}\text { Performance } \\
\text { Gains }\end{array}$ & Hedonic & Pleasure & $\begin{array}{l}\text { Semantic differential } \\
\text { scale }\end{array}$ \\
\hline
\end{tabular}




\section{Observational methodology}

3D Live has developed an observational method for carrying out evaluation trials in real-time tele-immersive environments. Tele-immersive systems pose challenges due to the need to coordinate and collect data from geographically distributed system components and users. A coordinated approach to these trials requires unified control over an experimental workflow to orchestrate participants and the ability to monitor and aggregate synchronized metric data from multiple sources. A central human coordinator provides executive control over the experimental process to support workflow execution and data gathering. Communication between the coordinator and local support teams was achieved using a shared, on-line workflow defining the process model and the ability to collect fine-grained notes on the status the game as it progressed. The process model contained all steps needed to consistently set-up, execute and tear-down the system under test including distributed interactions with remote users in a repeatable way.

In addition to the workflow management described above, a systematic approach to metric data collection was provided using an experiment data management system called the EXPERIMonitor (EM) developed as part of the EXPERIMEDIA project [19]. The EM is a web service and data visualisation tool that offers support for realtime automatic metric based data acquisition and aggregation from remotely connected sources. Selected technical components within the 3D-LIVE system were instrumented using the EM API to provide specific sets of QoS data during each game, live at run-time. The coordinator used the EM to step through an experiment based process where connected components (or 'EM clients') are managed through specific phases that perform coordinated set-up, live data collection and tear-down related activities. Orthogonal to the quantitative observations, qualitative data was gathered in the form of video recordings of participating users, and post-game user interviews. Video data and interview based feedback provided us with early, rapid insights into the overall QoE and their perceptions of system performance.

\section{$5 \quad$ Preliminary Results}

Initial trials of all three scenarios have been carried out, each of which involving a series of repeated evaluation runs (between 15 and 20 games were typical). The purpose of these trials was two-fold; first to act as verification of system behaviour when deployed in the real-world and the second to gather a preliminary data-set of QoS measures and actual UX from which a 'baseline' of system behaviour and accompanying UX could be defined. Our QoS data set covered a range of measurements mapped to interactivity, including graphics rendering; human motion capture and reconstruction; voice communications and environment reconstruction data. Inspection of some of the principal QoS criteria defined for 3D-LIVE has revealed useful insights. Spatial-temporal consistency is one such criterion in our UX model: it provides an indication of closeness of mapping between indoor and outdoor interactions.

Fig. 3. Average distance of real-world skier to virtual avatar 

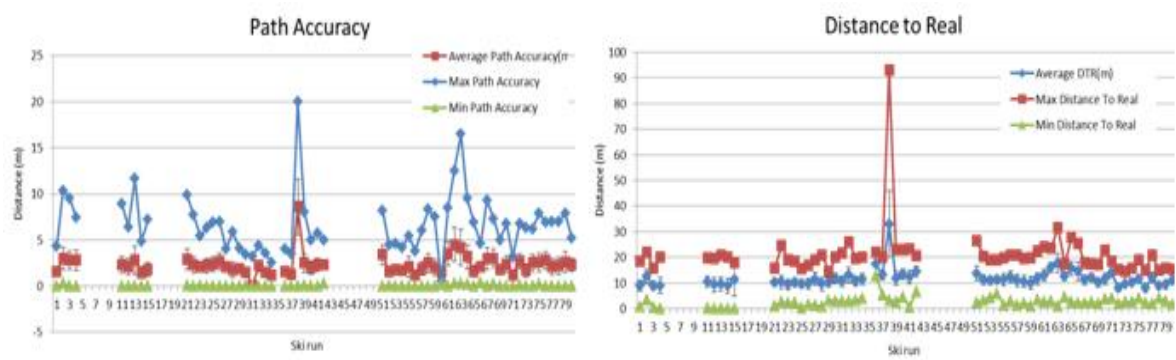

An example of spatial-temporal QoS data, from the skiing scenario, can be seen in Fig. 3. We show two sets of measures that relate the actual position of real-world skier and that of his avatar rendered to indoor users on the virtual slope. The path taken by the avatar representing the outdoor user is estimated using remote GPS data combined with a skiing motion simulation within the 3D-LIVE system. In the 'path accuracy' series we see the average deviation of the avatar's path from the actual position of the outdoor for each run. This data indicates that the path of is replicated virtually on average is $2.5 \mathrm{~m}$. We visualize the spatial consistency using a path representation shown below. A temporal analysis of the path data, shown in the 'distance to real' (DTR) series shows the distance between the outdoor skier's last known position on the real-world slope and the estimated position of his avatar. Here a wider discrepancy is evident $(10 \mathrm{~m})$ suggesting that update latency is impacting spatial accuracy over time; an average calculation of skiing speed at $10 \mathrm{~m} / \mathrm{s}$ suggests a delay of around one second.

In addition to collecting metrics reflecting technical performance, qualitative feedback was gathered from participants during trials. Here users were quick to identify issues that affected their overall UX; these included inaccuracies in movement reconstruction and update latencies (i.e. delays in voice communication). Interestingly, in both the skiing and jogging scenarios, the mapping between the physical movement of indoor users and their perceptions of their corresponding avatar behaviour was considered as unsatisfactory. In technically replicating the physi-

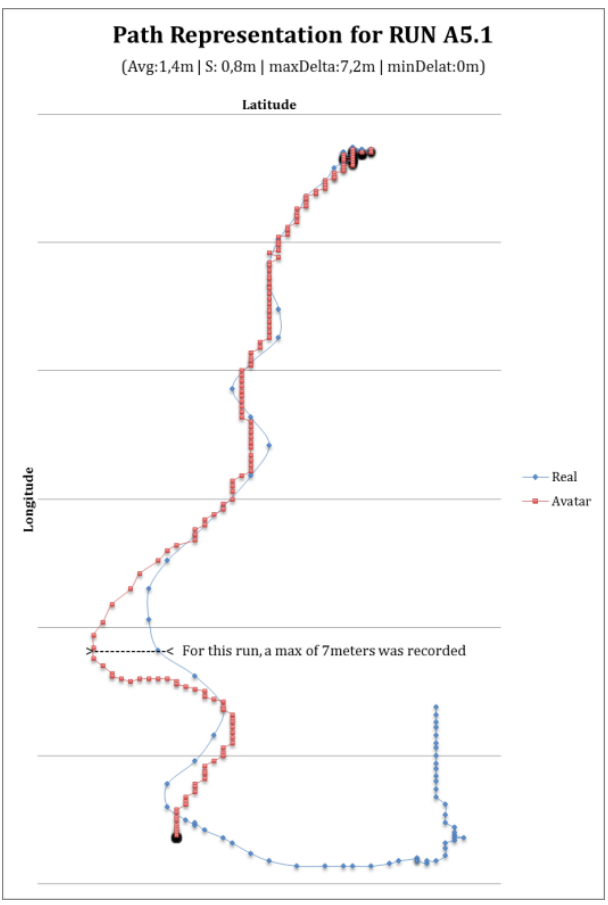
cal behaviour of users, a 1-to-1 mapping is applied as closely as possible in the virtual world - these avatar motions are displayed in the third person. However, in some cases the apparent changes to avatar motion (viewed at a third person distance) did not result in the 'expected' progress through the game (avatars were considered to be 
moving too slowly). This was mainly observed in the ski scenario, where the users were looking for a stronger link between their body pose on the simulator or the Wii balance board and the speed of their avatars. Overall the evaluations of the prototypes were enthusiastically received by users both formatively during co-creation and during summative trials. Early evaluation of the use of the technology was productive in that it revealed real-world performance and technical issues to be addressed as well as new ideas for future interaction design.

\section{Conclusion}

Our approach to design and evaluate complex tele-immersive systems combines usercentric co-creation and a distributed observation methodology. Using constructs from the IUX model, qualitative user input can be captured and used to drive design and implementation allowing for refinement of understanding of technical requirements and how UX can be enhanced. Qualitative and quantitative observations captured during trials have served to identify the aspects of actual UX that were considered important and a means by which system performance (QoS) can be understood and improved. Lessons learnt include an updated experiential model; changes and improvements to the 3D-LIVE interaction design; and better QoS and QoE metrics including a structured QoE questionnaire to provide formal validation of the experiential model. The lessons will be factored into our second phase of experimentation.

\section{$7 \quad$ Acknowledgements}

The authors would like to thank the staff of Schladming $2030 \mathrm{GmbH}$ (Austria) and Laval Golf, La Mayenne (France) for supporting our experimental work.

\section{$8 \quad$ References}

1. 3D Live Project http://3dliveproject.eu/wp/

2. Zhenyu Yang, Wanmin Wu, Klara Nahrstedt, Gregorij Kurillo, and Ruzena Bajcsy. 2010. Enabling multiparty 3D tele-immersive environments with ViewCast. ACM Transactions on Multimedia Computing, Communications, and Applications, 6(4): article 29.

3. Nakevska, M., Vos, C., Juarez, A., Hu, J., Langereis, G., Rauterberg, M. 2011. Using Game Engines in Mixed Reality Installations. In Entertainment Computing - ICEC 2011. Lecture Notes in Computer Science Volume 6972, 2011. pp456-459

4. Jean-Marie Normand, Bernhard Spanlang, Franco Tecchia, Marcello Carrozzino, David Swapp, and Mel Slater. 2012. Full body acting rehearsal in a networked virtual environment - a case study. Presence: Teleoper. Virtual Environ. 21, 2 (April 2012), 229-243.

5. Michael Gervautz, Dieter Schmalstieg, "Anywhere Interfaces Using Handheld Augmented Reality," Computer, vol. 45, no. 7, pp. 26-31, July, 2012

6. Chris Gauthier Dickey. 2010. Blending games, multimedia and reality. In Proceedings of the first annual ACM SIGMM conference on Multimedia systems (MMSys '10). ACM, 
New York, NY, USA, 209-214. DOI=10.1145/1730836.1730862 http://doi.acm.org/10.1145/1730836.1730862

7. Oshima, T., Sato K., Yamamoto, H., and Tamura, H. 1998. AR2 Hockey: A case study of Collaborative Augmented Reality. Virtual Reality Annual International Symposium, 1998. Proceedings., IEEE 1998

8. Vaïkkynen, P. et. al, 2001. Using exercise cycle as a haptic input device in a virtual environment, EGVE'01 Proceedings of the 7th Eurographics conference on Virtual Environments \\& 5th Immersive Projection Technology, pp. 229-235, 2001.

9. Mueller, F.F. and Agamanolis, S. 2005. Sports over a distance. Computers in Entertainment, vol. 3, no. 3, pp. 4-4, July 2005.

10. Göbel, S., Geiger, C., Heinze, C., and Marinos, D. 2010. Creating a virtual archery experience. In Proc. of The International Conference on Advanced Visual Interfaces (AVI '10), Rome, Italy, 2010.

11. Pallot, M., Eynard, R., Poussard, B., Christmann, O and Richir, S. 2013. Augmented sport: exploring collective user experience. In Proceedings of the Virtual Reality International Conference: Laval Virtual (VRIC '13). ACM, New York, NY, USA, , Article 4, 8 pages. DOI=10.1145/2466816.2466821 http://doi.acm.org/10.1145/2466816.2466821

12. Pallot, M., Trousse, B., Senach, B., and Scapin, D. 2010. Living Lab Research Landscape: From User Centred Design and User Experience towards User Cocreation. In Proceedings of the Living Lab Summer School, Paris, Cité des Sciences, August 2010.

13. Kim, S. J. J. 2012. A User Study Trends in Augmented Reality and Virtual Reality Research: A Qualitative Study with the Past Three Years of the ISMAR and IEEE VR Conference Papers, Ubiquitous Virtual Reality (ISUVR), 2012 International Symposium on, On page(s): 1 - 5

14. Gabbard, J.L., Swan, E. 2008. Usability Engineering for Augmented Reality: Employing User-Based Studies to Inform Design. In IEEE Transactions on Visualization and Computer Graphics. Vol. 14. No.3, MAY/JUNE 2008.

15. Rosson, M. B. and Carroll, J. M. 2002. Scenario-based design. In The human-computer interaction handbook, Julie A. Jacko and Andrew Sears (Eds.). L. Erlbaum Associates Inc., Hillsdale, NJ, USA 1032-1050.

16. Wu, W., Arefin, A., Rivas, R., Nahrstedt, K., Sheppard, R. and Yang, Z. 2009. Quality of experience in distributed interactive multimedia environments: toward a theoretical framework. In Proceedings of the 17th ACM international conference on Multimedia (MM '09). ACM, New York, NY, USA, 481-490. DOI=10.1145/1631272.1631338 http://doi.acm.org/10.1145/1631272.1631338

17. Doumanoglou, A., Alexiadis, D, Zarpalas, D., Daras, P. 2013. CERTH/ITI “Towards RealTime and Efficient Compression of Human Time-Varying-Meshes", IEEE Transactions on Circuits and Systems for Video Technology and 105th MPEG meeting, Jul-Aug 2013, Vienna.

18. Epstein, S. 2003. Cognitive-Experiential Self-Theory of Personality. In Millon, T. \& Lerner, M. J. (Eds), Comprehensive Handbook of Psychology, Vol. 5: Personality and Social Psychology (pp. 159-184). Hoboken, NJ, Wiley \& Sons.

19. Boniface, M., Osborne, D. S., Voulodimos, T., \& Murg, S. 2013. Technology Enablers for a Future Media Internet Testing Facility, NEM Summit 2013

20. Poussard, B., Richir, S., Vatjus-Anttila, J., Asteriadis, S., Zarpalas, D., Daras, P. 2014 3DLIVE: A Multi-Modal Sensing Platform Allowing Tele-Immersive Sports Applications, 22nd European Signal Processing Conference (EUSIPCO 2014), Lisbon, Portugal, 2-5 Sept. 2014 